\title{
Heparin modulation on hepatic nitric oxide synthase in experimental steatohepatitis
}

\author{
AMAL HASSANIN ${ }^{1}$, HALA ABDEL MALEK ${ }^{1}$ and DALIA SALEH ${ }^{2}$ \\ Departments of ${ }^{1}$ Clinical Pharmacology and ${ }^{2}$ Anatomy and Embryology, Faculty \\ of Medicine, Mansoura University, Mansoura 35516, Egypt
}

Received March 6, 2014; Accepted July 21, 2014

DOI: 10.3892/etm.2014.1963

\begin{abstract}
Nonalcoholic fatty liver disease (NAFLD) is considered to be a hepatic manifestation of metabolic syndrome, and has been etiologically associated with insulin resistance (IR). The histopathology of NAFLD ranges between simple steatosis and nonalcoholic steatohepatitis (NASH), with or without fibrosis. The aim of the present study was to examine the effect of heparin on steatohepatitis and hepatic-induced nitric oxide synthase (iNOS) expression in mice. Male mice were divided into four groups, which included the normal basal diet (control), high fat (HF) diet, HF diet + heparin (treatment group) and heparin control groups. After eight weeks from the initiation of the experiment, blood was collected and livers were harvested for biochemical analysis and histological studies. Serum levels of aspartate aminotransferase, alanine aminotransferase, hepatic triglyceride (TG) and hydroxyproline, as well as the IR, superoxide anion generation and mRNA expression of the hepatic iNOS enzyme were evaluated. Liver specimens were processed for histopathological and immunohistopathological evaluation. Heparin administration decreased the levels of the liver enzymes, IR, superoxide generation, hepatic TG, hydroxyproline and iNOS expression when compared with the HF diet group. These changes were associated with an improvement in inflammation and fibrosis observed via histopathological examination. Therefore, heparin treatment attenuates hepatic injury in steatohepatitis.
\end{abstract}

\section{Introduction}

Nonalcoholic fatty liver disease (NAFLD) occurs in individuals that have not consumed alcohol in amounts considered harmful to the liver. The disease is characterized by macrovesicular hepatic steatosis. There are two histological patterns of NAFLD: Fatty liver alone and steatohepatitis. Nonalcoholic

Correspondence to: Dr Hala Abdel Malek, Department of Clinical Pharmacology, Faculty of Medicine, Mansoura University, 24 El Gomhoria Street, Mansoura 35516, Egypt

E-mail: halamalek@live.com

Key words: heparin, fatty liver, inducible nitric oxide synthase, mice steatohepatitis (NASH) is defined as lipid accumulation in the liver with evidence of cellular damage, inflammation and varying degrees of scarring or fibrosis (1).

The mechanisms underlying the increase in hepatic fat accumulation are unclear and little information is available on the time course of the development of hepatic steatosis. The association between macrovesicular steatosis of the liver and inflammatory changes and fibrosis in obese individuals has been reported for several decades (2). The spectrum of this condition ranges between silent nonalcoholic fatty liver disease and chronic liver disease with complications. Animal models indicate that the liver may accumulate lipids within a few weeks or even a few days (3).

NAFLD is present in the majority of patients with metabolic risk factors, including obesity and diabetes. Insulin resistance (IR) is a key pathogenic factor of hepatic fat accumulation. NAFLD exacerbates hepatic IR and precedes glucose intolerance. Once hepatic steatosis occurs, additional factors, including oxidative stress, mitochondrial dysfunction and adipocytokines, may enhance hepatocellular damage (4).

NASH may progress to cirrhosis, particularly when features of bridging fibrosis are present. No definite therapy is currently available to reverse this pathological process (4). A number of studies have investigated the efficacy of medications for the treatment of NASH; the drugs studied include orlistat, metformin, vitamin E, lipid-lowering drugs, pentoxifylline and pioglitazone $(4,5)$. In addition, thrombin inhibitors have been considered as a treatment for liver fibrosis (6), and have been found to decrease the lung collagen involved in pulmonary fibrosis (7-8).

The current study aimed to examine the effects of heparin, a thrombin inhibitor, on hepatic injury in an experimental model of NASH and IR, via analyzing the effects on hepatic inducible nitric oxide synthase (iNOS) expression and superoxide generation.

\section{Materials and methods}

Drugs. Heparin sodium (Nile Pharma, Cairo, Egypt) was administered subcutaneously to the mice in the herparin groups at a dose of $10 \mu \mathrm{g} /$ day (8).

Animals. A total of 80 male BALB/c mice (weight, 20-23 g; age, 5-7 weeks) were obtained from the Animal Center of 
the Research Unit at the Faculty of Medicine of Mansoura University (Mansoura, Egypt). The experiment was performed in accordance with the Guide for the Care and Use of Laboratory Animals, and the experimental procedures were approved by the local Animal Care and Use Committee of Mansoura University (Mansoura, Egypt). The animals were housed in plastic cages with a 12-h light/dark cycle, with free access to food and water throughout the experimental period.

Experimental design. Following acclimatization for one week through consumption of a basal diet, the mice were divided into four groups $(n=20)$ and fed the following diets for eight weeks. Control group mice were fed a normal basal diet. The mice in the high fat (HF) diet group were fed a diet containing $71 \%$ energy from fat, $11 \%$ energy from carbohydrates and $18 \%$ energy from proteins (9). Mice in the HF diet + heparin-treated group were administered $10 \mu \mathrm{g}$ heparin sodium via daily subcutaneous injections, following the initial consumption of the HF diet for one week (second week overall) and for the remaining weeks (6-8). Finally, mice in the heparin control group were fed the normal basal diet, but administered heparin as previously described.

Specimen collection and staining. Eight weeks from the start of the experiment, mice were anesthetized with an intraperitoneal injection of sodium pentobarbital ( $50 \mathrm{mg} / \mathrm{kg}$ body weight). Blood samples were collected for biochemical analysis and the livers were harvested for iNOS gene expression, histological and immunohistochemical examinations.

Biochemical analysis. Serum levels of aspartate aminotransferase (AST) and alanine aminotransferase (ALT) were measured using an automated technique (Clinical Chemistry Analyzer), while serum glucose levels were measured using a OneTouch ${ }^{\circledR}$ Ultra $^{\circledR}$ Blood Glucose meter (LifeScan, Inc., Milpitas, CA, USA). In addition, serum insulin levels were measured with an enzyme-linked immunosorbent assay (ELISA) kit (Crystal Chem, Inc., Downers Grove, Chicago, IL, USA), according to the manufacturer's instructions. The level of hepatic triglyceride (TG) was measured using an enzymatic method (Sigma-Aldrich, St. Louis, MO, USA), according to the manufacturers' instructions (10), while the hepatic hydroxyproline content was estimated, as described in a study by Bergman and Loxley (11). Hepatic superoxide anion generation was also estimated using a previously described method (12). The gene expression levels of iNOS were determined in the livers of the aforementioned groups by semi-quantitative polymerase chain reaction (PCR). In addition, PCR was performed with the livers of a fifth group of mice $(n=12)$ that received high fat diet and subcutaneous injections of a standard nonselective iNOS inhibitor, NG-monomethyl-L-arginine (L-NMMA; Sigma-Aldrich), at a dose of $25 \mathrm{mg} / \mathrm{kg} /$ day (as with the administration of the heparin regimen) (13) to inhibit the iNOS synthase enzyme.

Total RNA was extracted from tissue specimens using a column technique, according to the manufacturer's instructions (Vivantis Technologies, Subang Jaya, Malaysia). In total, $1 \mu \mathrm{g}$ total RNA was reverse transcribed to cDNA using a Maxima First Strand cDNA Synthesis kit (Thermo Fisher Scientific, Ontario, Canada). A total of $2 \mu \mathrm{l} \mathrm{cDNA} \mathrm{was} \mathrm{ampli-}$ fied in a thermal cycler instrument (Arktik Thermal Cycler;
Thermo Fisher Scientific, Pittsburgh, PA, USA) with 25 pmol each primer pair, $10 \mathrm{mM}$ of Tris- $\mathrm{HCl}, \mathrm{pH} 8.3,50 \mathrm{mM}$ of $\mathrm{KCl}, 1.5 \mathrm{mM}$ of $\mathrm{MgCl}_{2}, 0.3 \mathrm{mM}$ of dNTP, $12.5 \mu \mathrm{l} 2 \mathrm{X} \mathrm{Taq}$ PCR Master mix (Qiagen, Inc., Valencia, CA, USA) and nuclease-free water in a total volume of $25 \mu \mathrm{l}$.

The primers designed for iNOS were 5'-CTCGGA ACTGTAGCACAGCA3' (sense) and 3'-GCACATCAAAGC GGCCATAG5' (antisense). $\beta$-actin was included as a reference gene, and had primer sequences of 5'-CAGGATTCCATA CCCAAGAAG-3' (sense) and 3'-AACCCTAAGGCCAAC CGTG-5' (antisense). The cycling parameters of the PCR amplification were as follows: Initial denaturation at $95^{\circ} \mathrm{C}$ for $5 \mathrm{~min}$, followed by 35 cycles of denaturation at $94^{\circ} \mathrm{C}$ for $30 \mathrm{sec}$, annealing at $60^{\circ} \mathrm{C}$ for $30 \mathrm{sec}$ and extension at $72^{\circ} \mathrm{C}$ for $45 \mathrm{sec}$, which was followed by a final extension at $72^{\circ} \mathrm{C}$ for $5 \mathrm{~min}$. PCR products were electrophoresed in $2 \%$ agarose gel (Vivantis Technologies) and visualized with an ethidium bromide stain (Sigma-Aldrich). A 100-bp DNA ladder (Vivantis Technologies) was applied to the first well to identify the molecular weight of the samples. Gel images were captured and analyzed with a Gel Documentation system (Bio-Rad, Hercules, CA, USA).

Histological procedure. Small pieces of liver were preserved in $10 \%$ formal saline for $\geq 48 \mathrm{~h}$, dehydrated in ascending grades of alcohol, cleared in xylene, embedded in paraffin and sectioned into $5-\mu \mathrm{m}$ thick sections. The sections were stained with hematoxylin and eosin to detect the histopathological changes, while Masson's trichrome and Sirius red stains were used to detect collagen. An $\alpha$-smooth muscle actin ( $\alpha$-SMA; Sigma-Aldrich, St.Louis, MO, USA) immunohistochemical stain was used to detect activated Ito cells.

Immunohistochemical procedure. A three-step indirect immunohistochemical technique was performed on $5-\mu \mathrm{m}$ formalin-fixed and paraffin-embedded sections. Antigen retrieval was achieved by heating the sections in a microwave oven at $560 \mathrm{~W}$ for $21 \mathrm{~min}$ in citrate buffer ( $\mathrm{pH} \mathrm{6.0)}$. The sections were subsequently treated with methanol containing $0.3 \%$ hydrogen peroxide for $15 \mathrm{~min}$ at room temperature in order to inactivate the endogenous peroxidase. Nonspecific binding of the secondary antibodies was minimized by incubation with $50 \%$ normal goat serum in phosphate-buffered saline (PBS) for $20 \mathrm{~min}$. Sections were incubated with appropriate primary antibodies ( $\alpha$-SMA 1A4; $1 / 50$ dilution) and diluted in PBS for $1 \mathrm{~h}$ in a humid chamber at room temperature. All rinsing procedures and serum dilutions were performed in PBS ( $\mathrm{pH}$ 7.2-7.4). The detection kit used was a Dako Cytomation LSAB ${ }^{\mathrm{TM}} 2$ System-HRP for rabbit and mouse (K0675; Dako Cytomation, Glostrup, Denmark). Positive reactions were visualized by applying DAB+ liquid (K3468; Dako, Carpinteria, CA, USA) for 5-10 min. Counterstaining with hematoxylin was conducted for $2 \mathrm{sec}$ and Aqueous Glycergel ${ }^{\circledR}$ Mounting medium (C563; Dako) was used to mount the stained sections.

Statistical analysis. Data are expressed as the mean \pm standard deviation. Analysis of variance followed by Tukey's post-hoc test was used to analyze the data, where $\mathrm{P} \leq 0.05$ was considered to indicate a statistically significant difference. All analyses 
Table I. Effect of heparin on various parameters in a NASH mouse model.

\begin{tabular}{|c|c|c|c|c|}
\hline Parameter & Control & HF diet & $\mathrm{HF}+$ heparin & Heparin control \\
\hline Serum insulin $(\mu \mathrm{U} / \mathrm{ml})$ & $10.83 \pm 1.37$ & $33.33 \pm 3.97^{\mathrm{a}}$ & $12.33 \pm 1.73^{\mathrm{b}}$ & $11.67 \pm 2.40^{\mathrm{b}}$ \\
\hline Serum glucose (mg/dl) & $102.50 \pm 6.40$ & $262.50 \pm 18.97^{\mathrm{a}}$ & $114.33 \pm 7.01^{\mathrm{a}, \mathrm{b}}$ & $105.17 \pm 8.02^{\mathrm{b}, \mathrm{c}}$ \\
\hline $\begin{array}{l}\text { Superoxide generation } \\
\text { (pmol/min/mg protein) }\end{array}$ & $24.83 \pm 6.63$ & $54.17 \pm 5.74^{\mathrm{a}}$ & $23.67 \pm 5.37^{\mathrm{b}}$ & $22.17 \pm 8.46^{\mathrm{b}}$ \\
\hline TG (mg/g) & $31.00 \pm 5.41$ & $131.67 \pm 7.16^{\mathrm{a}}$ & $43.83 \pm 9.90^{\mathrm{a}, \mathrm{b}}$ & $30.83 \pm 5.65^{\mathrm{b}, \mathrm{c}}$ \\
\hline Hydroxyproline ( $\mu \mathrm{mol} / \mathrm{mg}$ protein) & $2.27 \pm 1.51$ & $7.40 \pm 1.53^{\mathrm{a}}$ & $2.93 \pm 0.71^{\mathrm{b}}$ & $2.52 \pm 0.86^{\mathrm{b}}$ \\
\hline Serum ALT (U/l) & $28.00 \pm 4.73$ & $91.67 \pm 5.21^{\mathrm{a}}$ & $36.50 \pm 6.40^{\mathrm{a}, \mathrm{b}}$ & $27.33 \pm 4.46^{\mathrm{b}, \mathrm{c}}$ \\
\hline Serum AST (U/l) & $74.00 \pm 6.35$ & $154.50 \pm 12.63^{\mathrm{a}}$ & $78.00 \pm 9.81^{\mathrm{b}}$ & $73.67 \pm 9.96^{\mathrm{b}}$ \\
\hline HOMA & $2.76 \pm 0.49$ & $21.54 \pm 2.53^{\mathrm{a}}$ & $3.47 \pm 0.44^{\mathrm{b}}$ & $3.01 \pm 0.60^{\mathrm{b}}$ \\
\hline Body weight (g) & $19.50 \pm 5.30$ & $23.30 \pm 7.10$ & $21.50 \pm 6.30$ & $20.60 \pm 5.90$ \\
\hline
\end{tabular}

Results are expressed as the mean \pm standard deviation ( $\mathrm{n}=20$ per group). ${ }^{\mathrm{a}} \mathrm{P}<0.05$, vs. control; ${ }^{\mathrm{b}} \mathrm{P}<0.05$, vs. $\mathrm{HF}$; ${ }^{\mathrm{c}} \mathrm{P}<0.05$, vs. $\mathrm{HF}+\mathrm{heparin}$. TG, triglyceride; ALT, alanine transaminase; AST, aspartate aminotransferase; HF, high fat; NASH, nonalcoholic steatohepatitis; HOMA, Homeostatis Model Assessment.

were conducted using SPSS 11.0 software for Windows (SPSS, Inc., Chicago, IL, USA).

\section{Results}

Effects of heparin injection on serum levels of ALT and AST. When compared with the control group, the serum levels of ALT and AST were significantly higher in the HF diet group $(\mathrm{P} \leq 0.05)$. The increment of these parameters was significantly attenuated in the $\mathrm{HF}+$ heparin group $(\mathrm{P} \leq 0.05$; Table I) when compared with the HF diet group.

Effects of heparin injection on oxidative stress, hepatic TG and hepatic fibrosis. As shown in Table I, the levels of superoxide free radicals, hepatic hydroxyproline, TGs and serum insulin were significantly higher in the HF diet group when compared with the control group $(\mathrm{P} \leq 0.05)$. This increase was significantly reduced in the heparin-treated groups ( $\mathrm{HF}+$ heparin only; $\mathrm{P} \leq 0.05$ ) when compared with the HF diet group.

Effect of heparin on the mRNA expression of iNOS. Hepatic expression of iNOS was higher in the HF diet group compared with the control group. However, heparin treatment decreased iNOS expression when compared with the L-NMMA-treated HF diet group (Fig. 1).

Histopathological observations of NAFLD. In the control group, livers were composed of a number of lobules consisting of thin-walled central veins from which cords of hepatocytes radiated towards the lobule periphery and alternated with narrow irregular blood sinusoids. Around the periphery of each lobule, branches of the hepatic artery, hepatic portal vein and bile duct were present forming the portal tract. The hepatocytes were polygonal with a large vesicular nucleus (Fig. 2).

The most important histological characteristic of the HF diet group was the presence of severe macrovesicular and microvesicular steatosis in the hepatocytes. The steatosis was predominantly centrilobular and macrovesicular (Fig. 3). However, in certain sections, steatosis was observed throughout the lobule. Macrovesicular steatosis was characterized by large vacuoles occupying almost the entire cytoplasm and forcing the nucleus to the periphery of the cell. By contrast, microvesicular steatosis was characterized by multiple small lipid vacuoles with the nucleus located in the center of the cell (Figs. 3 and 4).

A number of animals developed NASH, where steatosis was accompanied by mild intralobular and periportal inflammation with prominent hepatocellular ballooning (Fig. 4). Certain sections also demonstrated fat cysts and lipogranulomas, glycogenated nuclei and cells containing Mallory's hyaline (Fig. 5). In the heparin-treated group, steatosis and necroinflammatory activity was minimal (Fig. 6).

Heparin treatment improved liver fibrosis. Masson's trichrome and Sirius red stains for collagen revealed minimal fibrosis in the control group (Fig. 7A). However, the extent of fibrosis increased in the HF diet group, particularly in the perisinusoidal area in zone 3 and in the periportal region. A number of animals also demonstrated bridging fibrosis and pericellular fibrosis (Fig. 7B, D and E). However, heparin treatment decreased the extent of fibrosis when compared with the HF diet group (Fig. 7C and F).

Immunohistochemistry. In the liver sections of the control mice, a positive reaction between the smooth muscle cells of the terminal and sublobular venous blood vessels was observed (Fig. 8A). In the HF diet group, Ito cells reacted positively with the $\alpha$-SMA antibody in the areas of fibrosis and in the walls of the central veins, as well as in the septal connective tissue (Fig. 8B). The livers of mice from the HF diet + heparin-treated group revealed fewer $\alpha$-SMA positive cells compared with the HF diet group (Fig. 8C).

\section{Discussion}

NAFLD is present in the majority of patients with metabolic and/or multiple risk factors, including obesity, diabetes and hypertension. There are two histological patterns of NAFLD: 

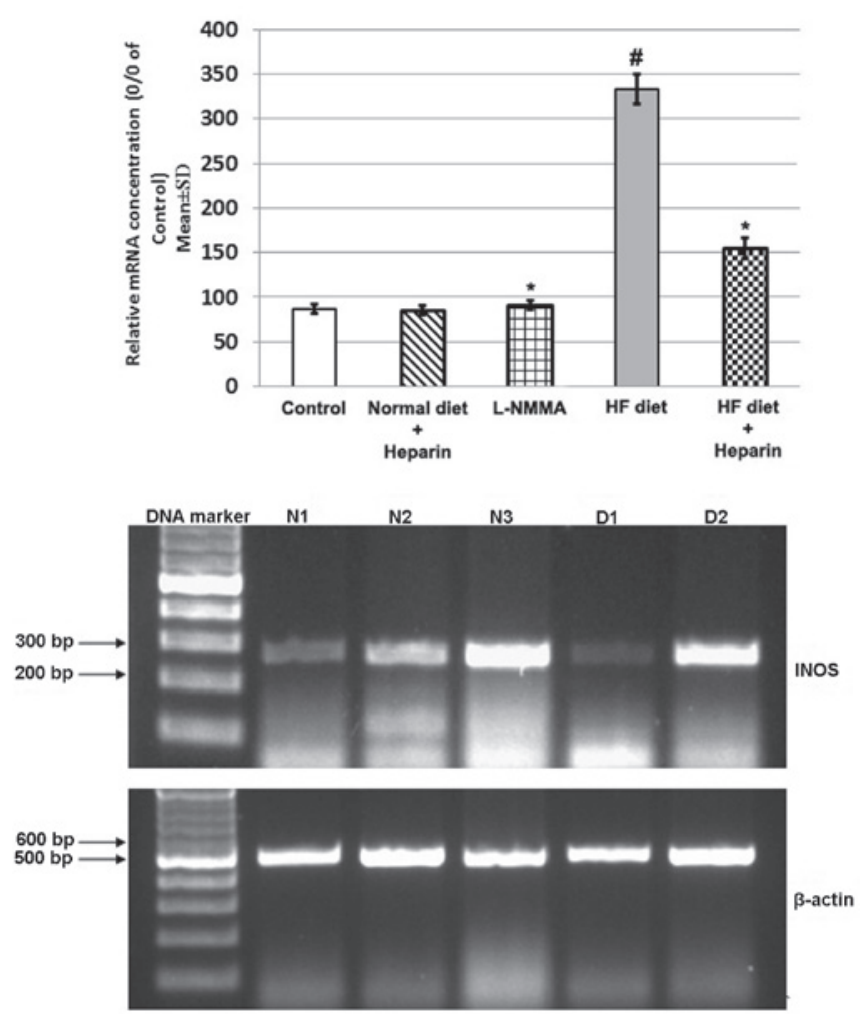

Figure 1. Effect of heparin administration on the mRNA expression levels of inducible nitric oxide synthase. Data are expressed as the mean \pm standard deviation ( $\mathrm{n}=12$ per group), and presented as the percentage of the control values following comparison by analysis of variance and Tukey's test. ${ }^{\#} \mathrm{P} \leq 0.05$ was considered to indicate a statistically significant difference in comparison to the control group. ${ }^{*} \mathrm{P} \leq 0.05$ was considered to indicate a statistically significant difference in comparison to the HF diet group. N1, control group; N2, control heparin group; N3, L-NMMA-treated high fat (HF) diet group; D1, HF diet (model) group; D2, heparin + HF diet group. L-NMMA, NG-monomethyl-L-arginine.

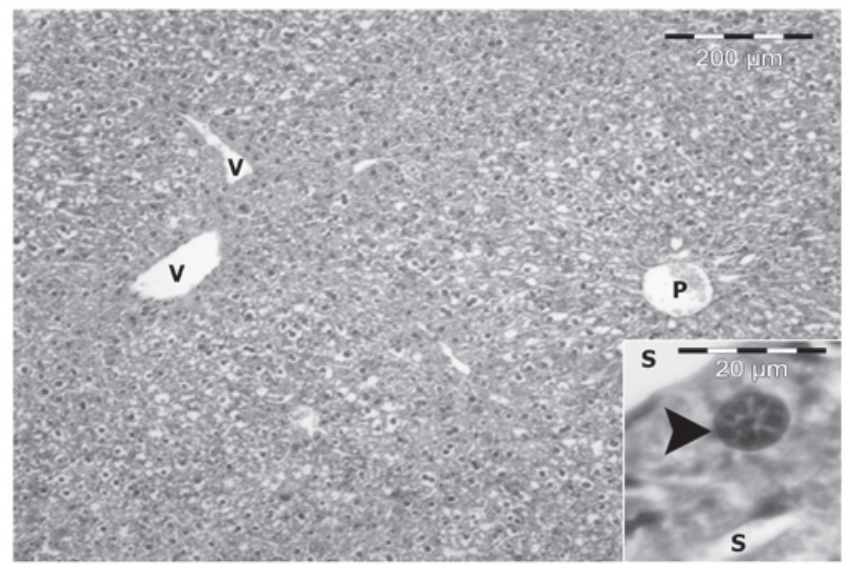

Figure 2. A photomicrograph (magnification, $\mathrm{x} 40$; hematoxylin and eosin stain) of the liver section of a control mouse showing one portal triad (P) and two central veins $(V)$ surrounded by hepatic cords and sinusoids. Inset: High magnification image (x400) of the hepatic cords showing a large polygonal hepatocyte with a vesicular nucleus (arrowhead) and blood sinusoids (S).

Fatty liver and steatohepatitis. NASH is defined as lipid accumulation associated with cell damage, inflammatory changes and fibrosis (1). NASH is a serious problem, with almost $25 \%$

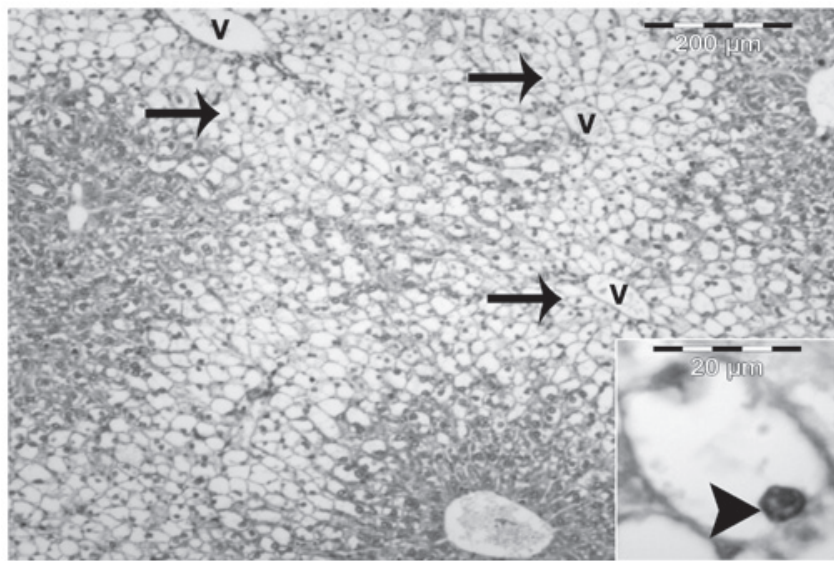

Figure 3. A photomicrograph (magnification, $x 40$; hematoxylin and eosin stain) of the liver section of a high fat-diet fed mouse showing nonalcoholic fatty liver disease in the form of severe macrovesicular (arrows) steatosis, which is present mainly in the perivenular region $(\mathrm{V})$ or acinar zone 3 . Inset: High magnification image ( $\mathrm{x} 400)$ showing a distended hepatocyte with a signet ring appearance (arrowhead). The cytoplasm is filled with a large empty vacuole and the nucleus is pushed to the periphery.

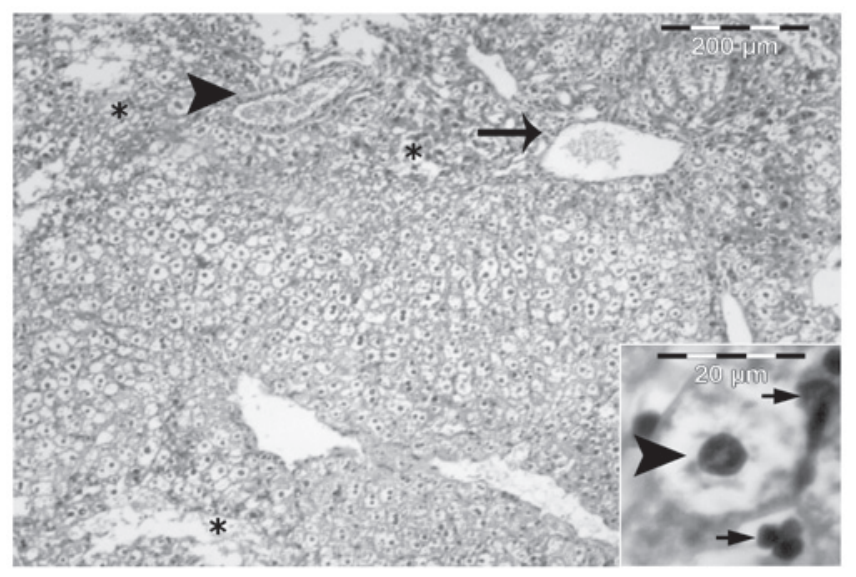

Figure 4. A photomicrograph (magnification, $x 40$; hematoxylin and eosin stain) of the liver section of a high fat-diet fed mouse showing nonalcoholic steatohepatitis in the form of mild periportal (arrow) and perivenular (arrowhead) inflammatory cell aggregation with panacinar macrovesicular steatosis. Areas of liver necrosis and destructed liver architecture are marked with an "'t. Inset: High magnification image ( $\mathrm{x} 400)$ showing the ballooning of a hepatocyte with rarefied cytoplasm (arrowhead) and inflammatory cells (small arrows) nearby.

of cases progressing to cirrhosis and subsequent complications of portal hypertension, liver failure and hepatocellular carcinoma (14).

In the present study, HF diet-fed mice were used to examine the effect of heparin on iNOS enzyme expression. The HF diet induced a pathology similar to human NASH, including steatosis, hepatic inflammation and fibrous tissue formation. Mice fed a HF diet for eight weeks also developed hyperglycemia and hyperinsulinemia, which mimicked the human metabolic syndrome process. These experimental protocols were selected in accordance with the study by Lieber et al (9), who reported that rats fed a HF diet ad libitum for three weeks demonstrated IR, as well as marked panlobular steatosis, increased hepatic lipid concentrations and oxidative damage.

The pathogenesis of steatohepatitis caused by metabolic factors is not fully understood; however, the induction of fat 


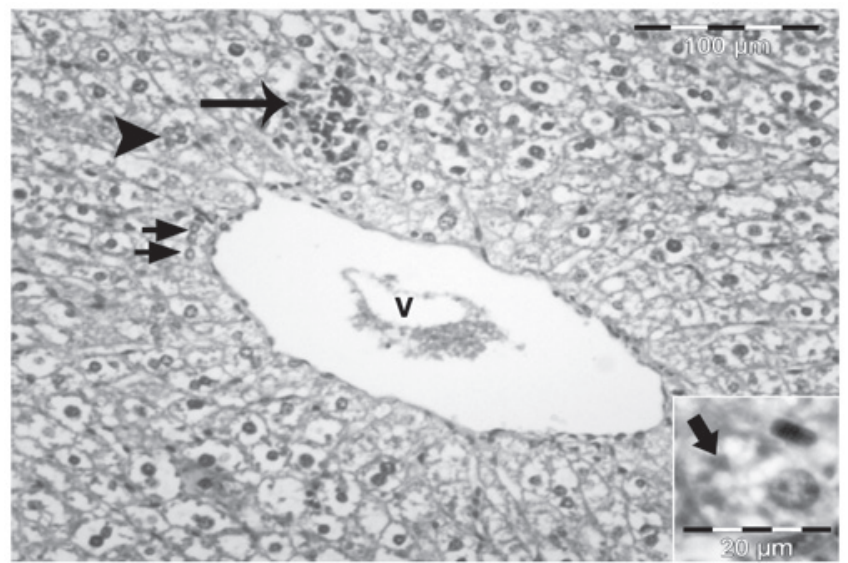

Figure 5. A photomicrograph (magnification, $x 40$; hematoxylin and eosin stain) of the liver section of a high fat-diet fed mouse showing nonalcoholic steatohepatitis in the form of focal necrosis with inflammatory cell aggregation (arrow) close to a dilated central vein (V). The lipogranuloma (arrowhead) and cells with glycogenated nuclei (short arrows) are marked. Inset: High magnification image (x400) showing a hepatocyte with Mallory's hyaline bodies in the form of eosinophilic and amorphous structures in the cytoplasm (thick arrow)

into the liver is essential. In experimental steatohepatitis caused by a lipogenic diet, hepatic steatosis represents the hepatic part of the metabolic syndrome disorder and is accompanied by IR and elevated TGs $(2,15)$.

The mechanism responsible for the increase in hepatic lipid accumulation is uncertain and little information is available regarding the time course of the development of hepatic steatosis. It has been hypothesized that fatty liver may result from increased delivery of free fatty acids (FFAs), impaired hepatic fatty acid oxidation and/or impaired synthesis or secretion of very low-density lipoproteins (16). Among these factors, the increased flow of FFAs to the liver is considered the most important (17). An increased rate of lipolysis and FFA abundance are the causes of oxidative stress in the liver. FFA oxidation generates oxidative stress and subsequent lipid peroxidation, which may be the mediator of subsequent inflammatory processes (18).

Oxidative stress is a mechanism of hepatocellular injury in steatohepatitis and is associated with the accumulation of lipid peroxidation products, elevation of proinflammatory cytokines and mitochondrial dysfunction (18-19). However, excess hepatic fat may lead to hepatic IR via the release of cytokines from Kupffer cells, leading to hepatocellular inflammation, reduced endothelial nitric oxide (eNO) signaling and the development of IR, which is a risk factor for liver fibrosis in steatohepatitis (20).

Excessive circulating FFAs exert deleterious effects on mitochondria and insulin signaling via protein kinase $\mathrm{C}$ activation and the inhibition of insulin receptor tyrosine kinase activity, leading to systemic IR (21).

The hepatic fibrosis observations in the present study, which resulted from TG accumulation and steatohepatitis, were consistent with those reported in the study by Poniachik et al (22), who concluded that the degree of steatosis in the liver biopsy is a risk factor for the development of fibrosis. In addition, Larter et al (23) revealed that in a nutritional model of steatohepatitis, accumulation of TGs occurs despite substantial suppression of lipogenesis and the induction of TG synthesis

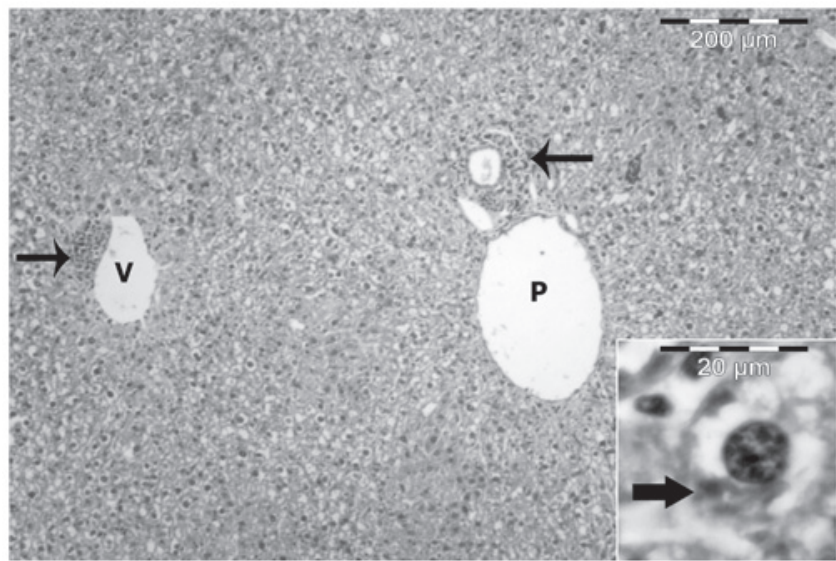

Figure 6. A photomicrograph (magnification, $x 40$; hematoxylin and eosin stain) of the liver section of a high fat-diet + heparin-treated mouse showing preserved liver architecture and a decrease in steatosis. Inflammatory cell aggregation (arrows) was observed around the portal area $(\mathrm{P})$ and the central vein $(\mathrm{V})$. Inset: High magnification image $(\mathrm{x} 400)$ showing a hepatocyte with Mallory's hyaline bodies in the form of eosinophilic and amorphous structures in the cytoplasm (thick arrow).

genes; these observations support the lipotoxicity mechanism involved in the liver injury component of metabolic syndrome.

Furthermore, the origin of the reactive oxygen species increase in NASH has been investigated. Reduced superoxide scavenging and glutathione replenishment may be involved in steatohepatitis, and antioxidant therapy may have a beneficial therapeutic effect for this purpose (24-25).

Administration of a HF diet in mice induces the proinflammatory activation of Kupffer cells, which is associated with reduced liver endothelial NOS activity. Kupffer cells produce the free radical, NO, from iNOS when they are stimulated by inflammatory cytokines or lipopolysaccharide (13-26).

Liu and Huang (27) supported the association between obesity-induced IR and the reduction in the eNO vascular content that causes a predisposition to increased endothelial inflammation, thrombosis and vasoconstriction. Furthermore, Tateya et al (28) revealed that a HF diet induces liver inflammation and IR. The authors determined that a reduction in eNO signaling leads to increased inflammation, which is inhibited by increasing eNOS activity.

Immunohistochemical examination of the livers of the mice in the HF diet group demonstrated increased expression levels of $\alpha$-SMA in the perisinusoidal stellate cells in areas of fibrosis around the portal spaces and fibrous septa. These results have been reported during the course of the development of hepatic fibrosis in humans and rats (29-32). Activated hepatic stellate cells synthesize tissue inhibitors of matrix metalloproteinase 1 and 2, which inhibit interstitial collagenase activity and contribute to the accumulation of extracellular matrix proteins; the latter of which is involved in the progression of chronic hepatic fibrosis (33-36).

NASH therapy remains under investigation and no definite therapy is currently available to reverse the cirrhotic pathological process (2). Previously, heparin was used in the management of vascular thrombosis. Heparin is a highly sulfated polysaccharide isolated from mast cells, which has been described as having antioxidant and antifibrotic activities. The drug has been used in the treatment of hepatic 
A

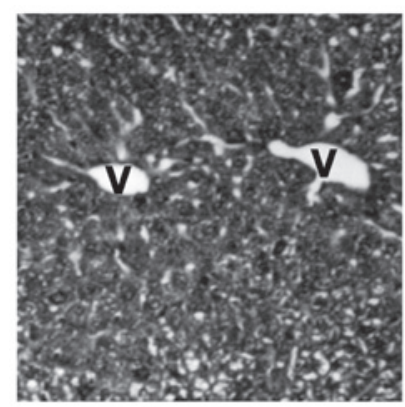

D

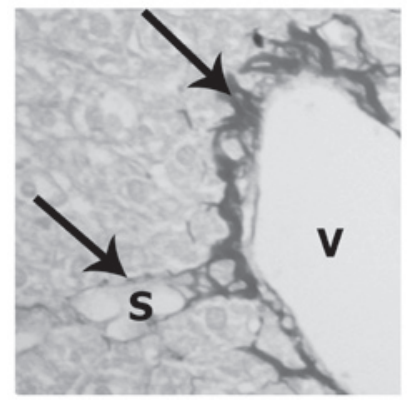

B

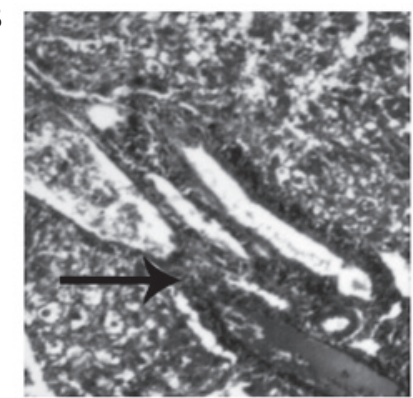

$\mathbf{E}$

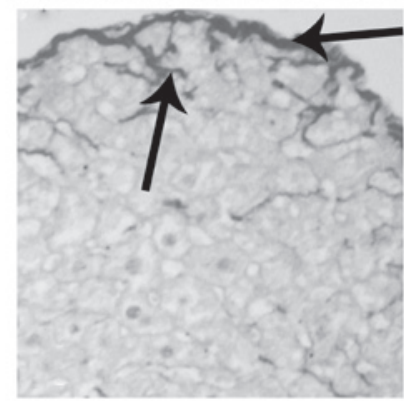

C

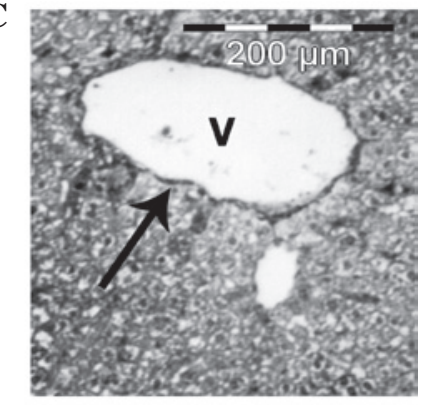

F

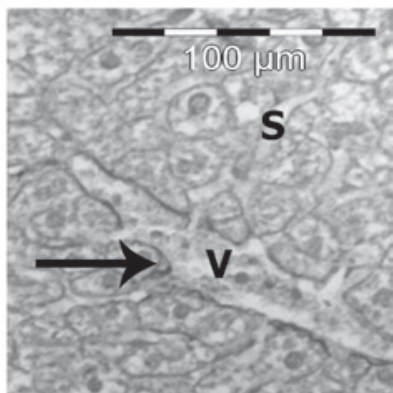

Figure 7. Photomicrographs of the mouse liver sections. (A) Normal control mouse liver showing normal arrangement of the central vein (V) and an intact parenchyma. (B) Liver section of high fat (HF) diet fed mice showing increased periportal fibrosis and development of a large septa (arrow) of connective tissue penetrating into the parenchyma. (C) Liver section of HF diet + heparin treated mice showing the central vein (V) with a decrease in perivenular fibrosis (arrow). (D) Liver section of high fat (HF) diet fed mice showing increased fibrosis (arrows) around the central vein (V) and blood sinusoids (S), (E) Liver section of high fat (HF) diet fed mice showing thickening of the liver capsule (arrows with a spread of fibrous tissue around the hepatocytes), and (F) Liver section of HF diet + heparin treated mice showing progressive breakdown of the fibrous septa (arrows) around the sinusoids (S) and the central vein (V). (A,B,C) Masson's trichrome stain; magnification, x40. (D,E,F) Siruis red stain; magnification, x100.

\section{A}

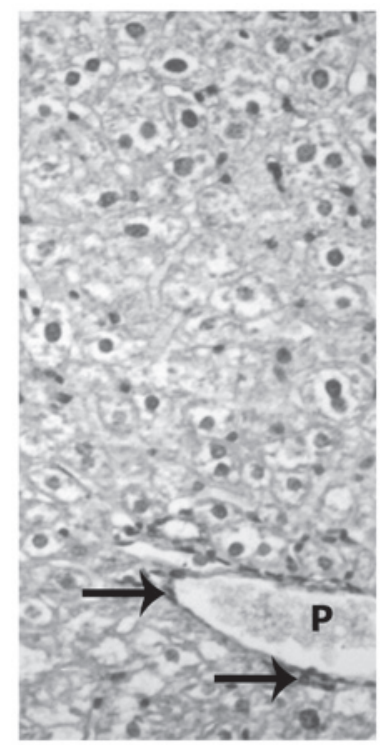

B

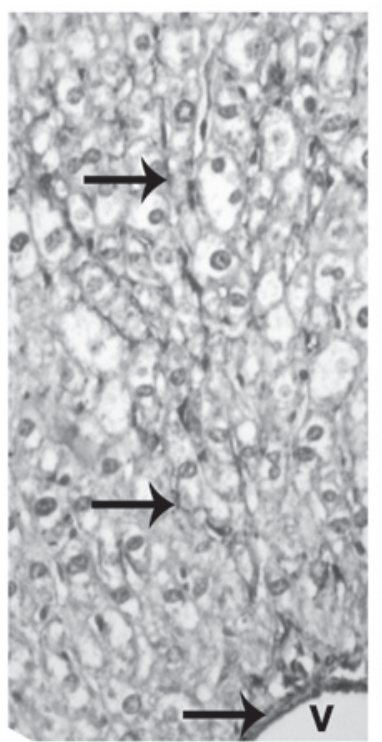

C

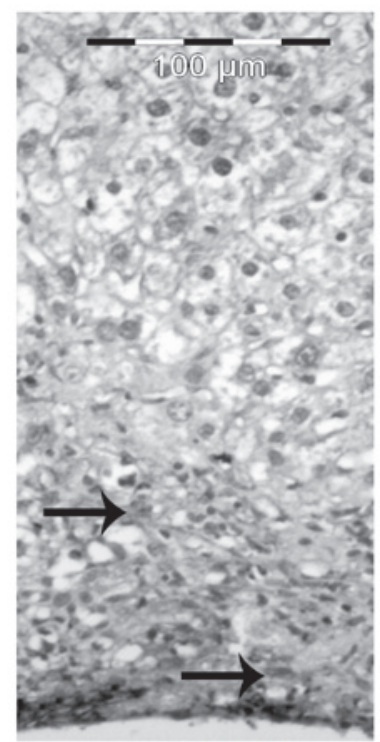

Figure 8. Photomicrographs (magnification, $\mathrm{x} 100$ ) of mouse liver sections using an $\alpha$-smooth muscle actin (SMA) immunoperoxidase stain. (A) Normal control mouse liver section showing $\alpha$-SMA positive cells (arrows) around the portal vein (P). (B) High fat (HF)-diet mouse liver section showing increased $\alpha$-SMA positive cells (arrows) in the wall of the central vein (V) and the perisinusoidal area. (C) HF-diet + heparin-treated mouse liver section showing few positive $\alpha$-SMA cells (arrows).

cholestasis and fibrosis $(6,7,37)$. In the present study, subcutaneous injections of heparin into mice fed a HF diet for eight weeks was shown to improve liver enzyme function, IR and hepatic fibrosis via the prevention of hepatic inflammation, improved IR and decreased signaling of iNOS. Heparin is known to have biological effects that are not associated with anticoagulant activity, including potential anti-inflammatory, immunomodulatory and antitumor effects. Certain studies have demonstrated that these actions may be hindered by the effect of heparin on hemostasis; however, not in the dosage used in the present study $(6,8,37)$.

The clinical benefit of heparin on chronic inflammatory disease has been previously studied. Heparin controls inflammation through trapping and neutralizing the inflammatory pathological proteins involved in the inflammatory process. This ability may be the result of its large polysaccharide 
chemical structure that binds to the different inflammatory mediators and cytokines, including antithrombin, heparin binding proteins and adhesion molecules (37). Mu et al concluded that heparin ameliorates lung injury and causes pulmonary vascular remodeling via the inhibition of iNOS expression (38-39). Furthermore, Ding et al (40) revealed that unfractionated heparin reduces inflammation and cytokine expression in endotoxemic mice. In addition, Saï et al (8) demonstrated that heparin attenuates low-dose streptozotocin-induced immune diabetes in mice in a dose similar to the dose used in the present study, but for a period of almost three weeks, as well as inhibiting the $\beta$-cell binding of T-splenocytes in vitro. The authors hypothesized that heparin at this dosage exhibits a variety of functions beyond an anticoagulant effect, without inducing the side effect of blood loss.

Tokuyama et al (41) revealed that diabetic patients who were administered insulin lispro and heparin treatment achieved improved glycemic control without any adverse effects. In addition, Shah and Shah demonstrated that heparin preserves hepatic function and reduces the severity of hepatic fibrogenesis following six weeks of treatment. Thus, heparin inhibits thrombin in vivo and in vitro. Thrombin is responsible for stimulating the proliferation of hepatic stellate cells (6). It interacts with specific cellular upregulated receptors that are located not only in the liver, but also in the lungs, resulting in the accumulation of collagen and fibrosis. Heparin is involved in the inhibition of the coagulation system and prevents acute liver injury from a hepatotoxic dose of lipopolysaccharide $(6,42)$.

In conclusion, heparin administration improves the liver histopathology of mice receiving a HF diet. This effect occurs via the downregulation of the iNOS gene and the suppression of hepatic TG content. The anti-inflammatory mechanism of heparin should be determined in future studies.

\section{Acknowledgements}

The authors thank the Experimental Medical Research Center (Faculty of Medicine, Mansoura University) for their significant contribution to the experimental care and biochemical analysis, and the Nile Center for Research (in particular Dr Thoria Medhat and her supervisor Dr Mahmoud Zakaria) for assistance with semi-quantitative PCR.

\section{References}

1. Brunt EM, Neuschwander-Tetri BA, Oliver D, Wehmeier KR and Bacon BR: Nonalcoholic steatohepatitis: histologic features and clinical correlations with 30 blinded biopsy specimens. Hum Pathol 35: 1070-1082, 2004.

2. Marchesini G, Brizi M, Morselli-Labate AM, Bianchi G, Bugianesi E, McCullough AJ, Forlani G and Melchionda N: Association of nonalcoholic fatty liver disease with insulin resistance. Am J Med 107: 450-455, 1999.

3. Diehl AM: Lessons from animal models of NASH. Hepatol Res 33: 138-144, 2005.

4. Kita Y, Takamura T, Misu H, Ota T, Kurita S, Takeshita Y, Uno M, Matsuzawa-Nagata N, et al: Metformin prevents and reverses inflammation in a non-diabetic mouse model of nonalcoholic steatohepatitis. PLoS One 7: e43056, 2012.

5. McCullough AJ: Thiazolidinediones for nonalcoholic steatohepatitis - promising but not ready for prime time. N Engl J Med 355: 2361-2363, 2006 .
6. Shah B and Shah G: Antifibrotic effect of heparin on liver fibrosis model in rats. World J Gastrointest Pharmacol Ther 3: 86-92, 2012.

7. Howell M, Mohun TJ and Hill CS: Xenopus Smad3 is specifically expressed in the chordoneural hinge, notochord and in the endocardium of the developing heart. Mech Dev 104: 147-150, 2001.

8. Saï P, Pogu S and Ouary M: Heparin attenuates low-dose streptozotocin-induced immune diabetes in mice and inhibits the beta-cell binding of T-splenocytes in vitro. Diabetologia 34: 212-217, 1991.

9. Lieber CS, Leo MA, Mak KM, Xu Y,Cao Q, Ren C,Ponomarenko A and Decarli LM: Model of nonalcoholic steatohepatitis. Am J Clin Nutr 79: 502-509, 2004.

10. Bligh EG and Dyer WJ: A rapid method of total lipid extraction and purification. Can J Biochem Physiol 37: 911-917, 1959.

11. Bergman M and Loxley R: The determination of hydroxyproline in urine hydrolysates. Clin Chim Acta 27: 347-349, 1970.

12. Wang HD, Pagano PJ, Du Y, Cayatte AJ, Quinn MT, Brecher P and Cohen RA: Superoxide anion from the adventitia of the rat thoracic aorta inactivates nitric oxide. Circ Res 82: 810-818, 1998.

13. Thiemermann C, Ruetten H, Wu CC and Vane JR: The multiple organ dysfunction syndrome caused by endotoxin in the rat: attenuation of liver dysfunction by inhibitors of nitric oxide synthase. $\mathrm{Br}$ J Pharmacol 116: 2845-2851, 1995.

14. Ekstedt M, Franzén LE, Mathiesen UL, Thorelius L, Holmqvist M, Bodemar $\mathrm{G}$ and Kechagias S: Long-term follow-up of patients with NAFLD and elevated liver enzymes. Hepatology 44: 865-873, 2006.

15. Chitturi S and Farrell GC: Etiopathogenesis of nonalcoholic steatohepatitis. Semin Liver Dis 21: 27-41, 2001.

16. Jansen PL: Non-alcoholic steatohepatitis. Eur J Gastroenterol Hepatol 16: 1079-1085, 2004.

17. McClain CJ, Mokshagundam SP, Barve SS, et al: Mechanisms of non-alcoholic steatohepatitis. Alcohol 34: 67-79, 2004.

18. Bonnefont-Rousselot D, Ratziu V, Giral P, Charlotte F, Beucler I, and Poynard T; Lido Study Group: Blood oxidative stress markers are unreliable markers of hepatic steatosis. Aliment Pharmacol Ther 23: 91-98, 2006

19. Albano E: Free radical mechanisms in immune reactions associated with alcoholic liver disease. Free Radic Biol Med 32: 110-114, 2002

20. Kim F, Pham M, Maloney E, Rizzo NO, Morton GJ, Wisse BE, Kirk EA, Chait A and Schwartz MW: Vascular inflammation, insulin resistance, and reduced nitric oxide production precede the onset of peripheral insulin resistance. Arterioscler Thromb Vasc Biol 28: 1982-1988, 2008.

21. Guilherme A, Virbasius JV, Puri V and Czech MP: Adipocyte dysfunctions linking obesity to insulin resistance and type 2 diabetes. Nat Rev Mol Cell Biol 9: 367-377, 2008.

22. Poniachik J, Mancilla C, Contreras J, Csendes A, Smok G, Cavada G, Rojas J, et al: Obesity: risk factor for steatohepatitis and hepatic fibrosis. Rev Med Chil 130: 731-736, 2002 (In Spanish).

23. Larter CZ, Yeh MM, Haigh WG, Williams J, Brown S, Bell-Anderson KS, Lee SP and Farrell GC: Hepatic free fatty acids accumulate in experimental steatohepatitis: role of adaptive pathways. J Hepatol 48: 638-647, 2008.

24. Laurent A, Nicco C, Tran Van Nhieu J, Borderie D, Chéreau C, Conti F, et al: Pivotal role of superoxide anion and beneficial effect of antioxidant molecules in murine steatohepatitis. Hepatology 39: 1277-1285, 2004.

25. von Montfort C, Matias N, Fernandez A, Fucho R, Conde de la Rosa L, Martinez-Chantar ML, et al: Mitochondrial GSH determines the toxic or therapeutic potential of superoxide scavenging in steatohepatitis. J Hepatol 57: 852-859, 2012.

26. Böhm T, Berger H, Nejabat M, Riegler T, Kellner F, Kuttke M, Sagmeister S, Bazanella M, et al: Food-derived peroxidized fatty acids may trigger hepatic inflammation: a novel hypothesis to explain steatohepatitis. J Hepatol 59: 563-570, 2013.

27. Liu VW and Huang PL: Cardiovascular roles of nitric oxide: a review of insights from nitric oxide synthase gene disrupted mice. Cardiovasc Res 77: 19-29, 2008

28. Tateya S, Rizzo NO, Handa P, et al: Endothelial NO/cGMP/VASP signaling attenuates Kupffer cell activation and hepatic insulin resistance induced by high-fat feeding. Diabetes 60: 2792-2801, 2011.

29. Friedmann SL: Molecular regulation of hepatic fibrosis, an integrated cellular response to tissue injury. J Biol Chem 275: 2247-2250, 2000.

30. Boisclair J, Doré M, Beauchamp G, Chouinard L and Girard C: Characterization of the inflammatory infiltrate in canine chronic hepatitis. Vet Pathol 38: 628-635, 2001. 
31. Bataller R and Brenner D: Liver fibrosis. J Clin Invest 115 209-218, 2005.

32. Mekonnen GA, Ijzer J and Nederbragt $\mathrm{H}$ : Tenascin-C in chronic canine hepatitis: immunohistochemical localization and correlation with necro-inflammatory activity, fibrotic stage, and expression of alpha-smooth muscle actin, cytokeratin 7, and $\mathrm{CD}^{+}$cells. Vet Pathol 44: 803-813, 2007.

33. Bedossa P and Paradis V: Liver extracellular matrix in health and disease. J Pathol 200: 504-515, 2003.

34. Guyot C, Lepreux S, Combe C, Doudnikoff E, Bioulac-Sage P, Balabaud $\mathrm{C}$ and Desmoulière A: Hepatic fibrosis and cirrhosis: the (myo)fibroblastic cell subpopulations involved. Int J Biochem Cell Biol 38: 135-151, 2006.

35. Hinz B, Phan SH, Thannickal VJ, Galli A, Bochaton-Piallat M and Gabbiani G: The myofibroblast: one function, multiple origins. Am J Pathol 170: 1807-1816, 2007.

36. Ijzer J, Roskams T, Molenbeek RF, Ultee T, Penning LC, Rothuizen $\mathrm{J}$ and van den Ingh TS: Morphological characterization of portal myofibroblasts and hepatic stellate cells in the normal dog liver. Comp Hepatol 5: 7, 2006.

37. Lever R and Page CP: Non-anticoagulant effects of heparin: an overview. Handb Exp Pharmacol 207: 281-305, 2012.
38. Mu E, Ding R, An X, Li X, Chen S and Ma X: Heparin attenuates lipopolysaccharide-induced acute lung injury by inhibiting nitric oxide synthase and TGF- $\beta /$ Smad signaling pathway. Thromb Res 129: 479-485, 2012.

39. Horstman DJ, Fischer LG, Kouretas PC, Hannan RL and Rich GF: Role of nitric oxide in heparin-induced attenuation of hypoxic pulmonary vascular remodeling. J Appl Physiol (1985) 92: 2012-2018, 2002

40. Ding R, Zhao D, Guo B, Zhang Z and Ma X: Treatment with unfractionated heparin attenuates coagulation and inflammation in endotoxemic mice. Thromb Res 128: e160-e165, 2011.

41. Tokuyama Y, Nozaki O and Kanatsuka A: A patient with subcutaneous-insulin resistance treated by insulin lispro plus heparin. Diabetes Res Clin Pract 54: 209-212, 2001.

42. Fiorucci S, Antonelli E, Distrutti E, Severino B, Fiorentina R, Baldoni M, et al: PAR1 antagonism protects against experimental liver fibrosis. Role of proteinase receptors in stellate cell activation. Hepatology 39: 365-375, 2004. 